Editorial

\title{
Recent Advances in Nanomaterials for Environmental Detection and Remediation
}

\author{
Jumin Hao, ${ }^{1}$ Qichen Wang, ${ }^{2}$ Jianfeng Zhang, ${ }^{3}$ Lili He, ${ }^{4}$ and Liping Qiu \\ ${ }^{1}$ Agiltron Inc., 15 Presidential Way, Woburn, MA 01801, USA \\ ${ }^{2}$ Micro Stamping Corporation, Somerset, NJ 08873, USA \\ ${ }^{3}$ School of Environmental and Municipal Engineering, Xi'an University of Architecture and Technology, Xian, Shanxi 710055, China \\ ${ }^{4}$ Department of Food Science, University of Massachusetts Amherst, Amherst, MA 01003, USA \\ ${ }^{5}$ School of Civil Engineering and Architecture, University of Jinan, Jinan, Shandong 250022, China
}

Correspondence should be addressed to Jumin Hao; jmhao0753@gmail.com

Received 31 May 2015; Accepted 31 May 2015

Copyright (c) 2015 Jumin Hao et al. This is an open access article distributed under the Creative Commons Attribution License, which permits unrestricted use, distribution, and reproduction in any medium, provided the original work is properly cited.

Environmental detection and remediation have been substantially benefiting from progress in nanomaterials and nanotechnologies in the past decades. Combining the nanomaterials and analytical techniques is very attractive to develop simple, sensitive, inexpensive, and miniaturized devices for field use for the detection of a variety of environmental contaminants. The nanomaterials also have great potential in advancing the treatment technologies for contaminated water, air, soil, and sediment. As experienced experts in the related areas for many years, we were invited to organize an editorial board to create a special issue to provide environmental research community with a forum to share innovative ideas and to present an up-to-date account of advancement in these fields.

Specifically, this special issue aims to study (a) preparation and application of various nanomaterials and nanostructures, made from metals and metal (hydro)oxides, carbon and fullerene derivatives, polymers, biomacromolecules, and their composites, for environmental detection and remediation; (b) identification and quantification of chemical, biological, and medical agents/toxins in various media (air, water, soils, and foods) based on nanomaterials and nanosensors; (c) sensing principles and mechanisms of nanomaterials based surface plasmon resonance (SPR), surface enhanced Raman scattering (SERS), fluorescence, colorimetry, and electrical and electrochemical sensing; (d) water/wastewater treatment, air quality control, soil and sediment remediation using nanomaterials, nanocatalysts, and nanomaterialsdoped membrane technology; (e) adsorption, degradation, and removal mechanisms of the environmental contaminants over nanoadsorbents and nanocatalysts and nanotechnologybased membrane separation, filtration processes, and photodegradation; (f) fate and transport and determination methods of engineered nanomaterials in environmental media; (g) demonstration and test of portable/handheld devices or prototypes for field use for pollutants detection and environmental remediation. The special issue consists of five research papers selected from many submissions. While they may not fully cover the topics mentioned above, these five papers represent the rich and many-faceted knowledge.

A paper entitled "Detection of Nanoscale Soil Organic Matter by Middle Infrared Spectrum for Forensic Science" by Y. Liu et al. evidenced that soil can provide significant assistance to forensic science by determining nanoscale soil organic matters (NSOMs) with middle infrared spectrum. In the study, eighteen soils sampled from different locations were investigated and results showed that the constituents and contents of NSOMs in the samples were dramatically different, and a NSOM fingerprint for each sample was drawn based on these characteristics. This suggests that a national or global NSOM fingerprint database could be rapidly established by the one-step middle infrared spectrum analysis for 
different soil samples, which will be helpful in determining crime scenes by comparing the middle infrared spectrum of forensic soil with the NSOMs fingerprint database.

In another paper entitled "Micro- to Nanoscale Morphologies and Chemical Components of Soils Investigated by SEM-EDS for Forensic Science," Y. Liu et al. further demonstrated that Scanning Electron Microscope-Energy Dispersive Spectrometer (SEM-EDS) is a useful tool to evaluate homogeneities and diameters of the soils at nanoscale. The mass fraction and molar percentages of nine inorganic elements in all samples were analyzed by EDS. Oxygen and silicon showed the highest content in all of these samples. However, different samples exhibited their own characteristic elements, which can help in discriminating them from other samples. In this regard, SEM-EDS-based homogeneity and element analysis might be used as a fast and reliable technique for the soil criminological analysis.

A contribution to this special issue, entitled "Effects of Nanoscale Carbon Black Modified by $\mathrm{HNO}_{3}$ on Immobilization and Phytoavailability of $\mathrm{Ni}$ in Contaminated Soil" by J. Cheng et al., reported a surface-modified nanoscale carbon black (MCB) as Ni adsorbent in contaminated soil prepared by oxidizing the carbon black with $65 \% \mathrm{HNO}_{3}$. The surface properties of the adsorbent were characterized with zeta potential analysis, scanning electron microscopy (SEM), and Fourier transform infrared spectroscopy (FTIR). The authors investigated adsorption performance of $\mathrm{Ni}^{2+}$ by $\mathrm{MCB}$ and examined the effects of MCB on the DTPA-extractable $\mathrm{Ni}^{2+}$ in soil, $\mathrm{Ni}^{2+}$ uptake of ryegrass shoot, and growth of ryegrass. Results indicated that MCB possessed enhanced sorption capacity for Ni compared to $\mathrm{CB}$ and could be applied in the in situ immobilization, remediation of heavy metal contaminated saline-alkali soils, and phytoavailability.

Recently, PM2.5 (particulate matter with diameter of 2.5 microns or less) has become a major health hazard from the polluted air in many cities in China. The study by X. Li and Y. Gong in the paper entitled "Design of Polymeric Nanofiber Gauze Mask to Prevent Inhaling PM2.5 Particles from Haze Pollution" reported a polysulfone nanofiber as mask filtration material by electrospinning technique. The nanofiber mask material was characterized by SEM, air permeability test, and PM2.5 trapping experiment. The results indicate that nanofiber mask material can efficiently filter out the PM2.5 particles and simultaneously preserve a good breathability. In this regard, the nanofiber based material would be made into the comfortable and effective mask for preventing inhaling the harmful particles in haze air pollution. The nanofiber masks could be developed to commercial available masks in the future.

Finally, the contribution entitled "Effect of Modifying Prosthetic Socket Base Materials by Adding Nanodiamonds" by L. Ma et al. demonstrated that adding a predetermined amount of nanodiamonds (between 0.1 and $1.0 \mathrm{wt} . \%$ ) to the prosthetic socket base material can increase the glass transition temperature and improve the mechanical properties of the cured base material. More importantly, the authors' investigation indicated that the nanodiamond addition to the system could reduce the amount of unreacted volatile monomer and decrease the adverse effects of any unreacted volatile monomer on the working environment. This provides a potential for improving the method and safety for the processing and modification of prosthetic socket base materials.

Compiling these papers, we hope to enrich our readers and researchers with respect to the most recent progresses in the field of the nanomaterials for environmental detection and remediation.

\section{Acknowledgments}

We, the guest editors, would like to appreciate all the authors for their contributions and all reviewers for their time and valuable comments to improve the quality of these papers.

Jumin Hao

Qichen Wang

Jianfeng Zhang

Lili He

Liping Qiu 

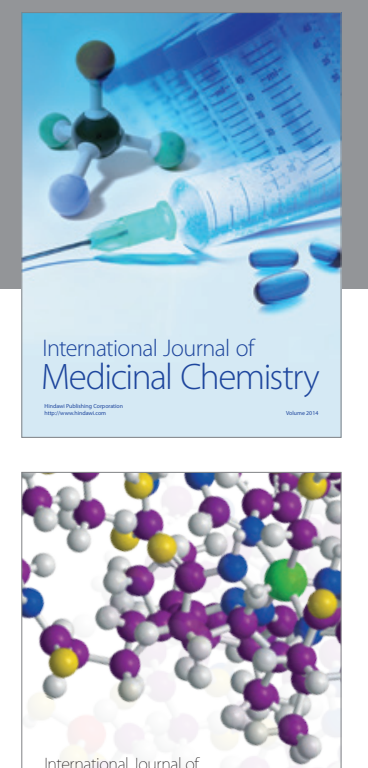

\section{Carbohydrate} Chemistry

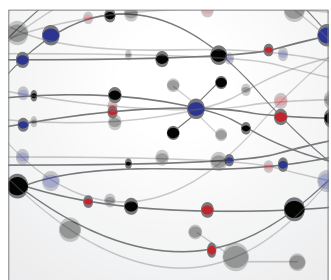

The Scientific World Journal
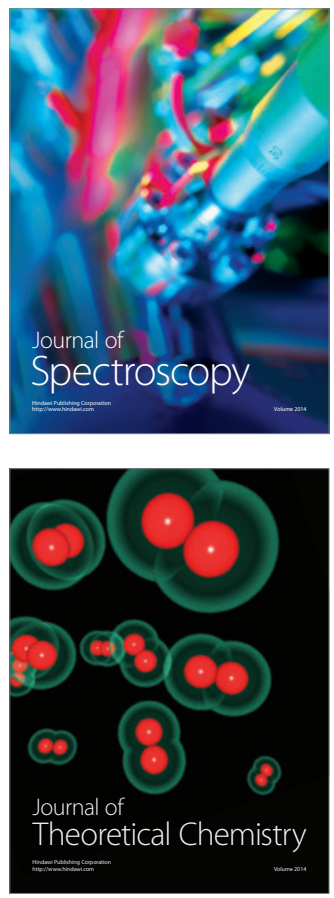
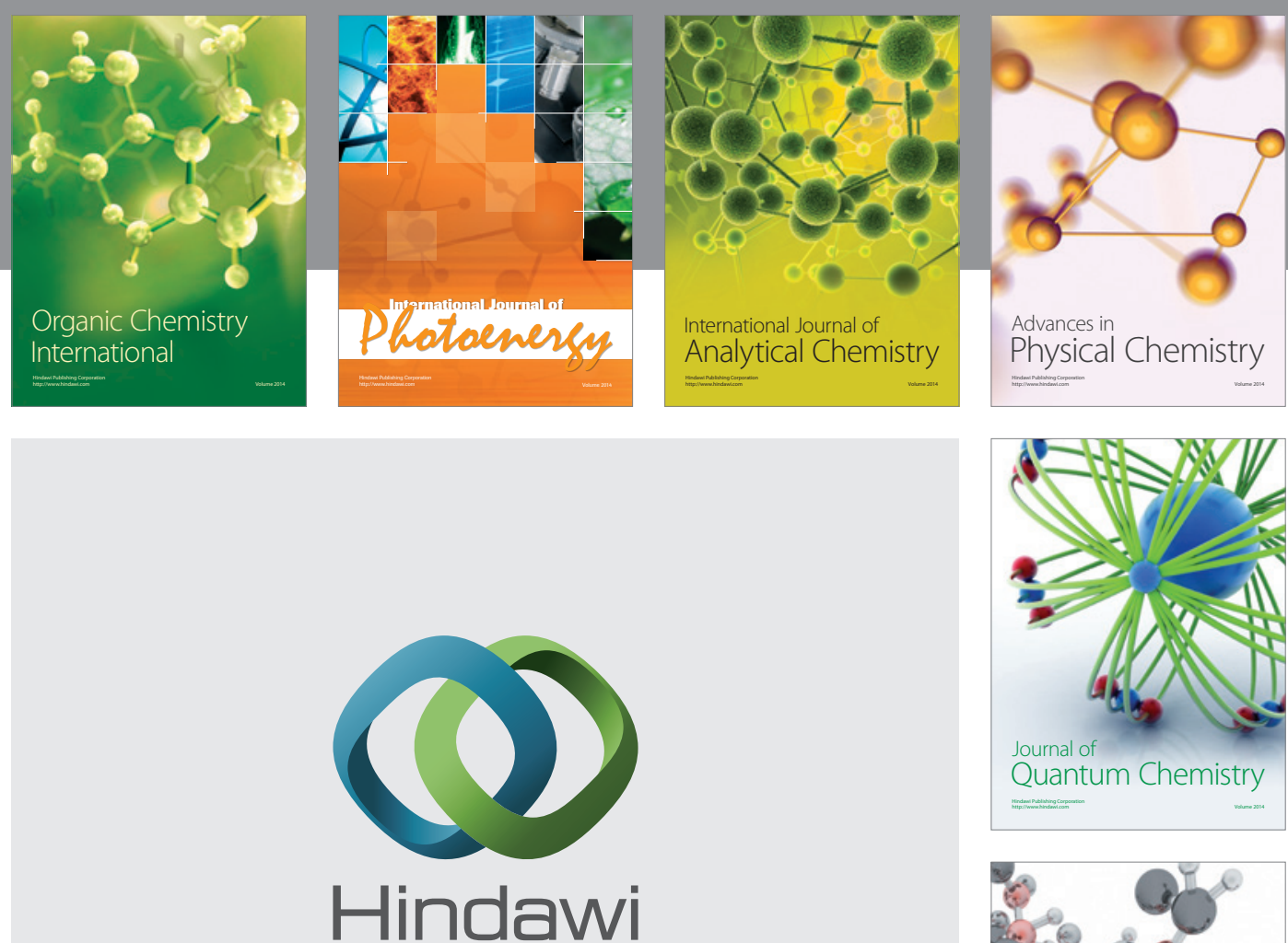

Submit your manuscripts at

http://www.hindawi.com

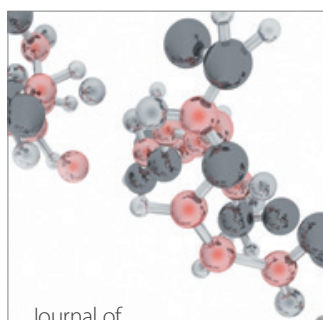

Analytical Methods

in Chemistry

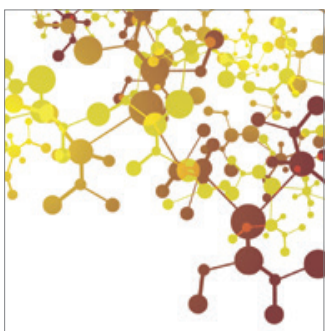

Journal of

Applied Chemistry

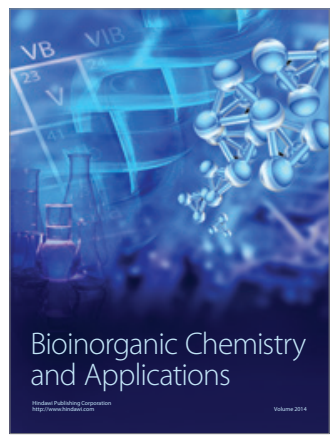

Inorganic Chemistry
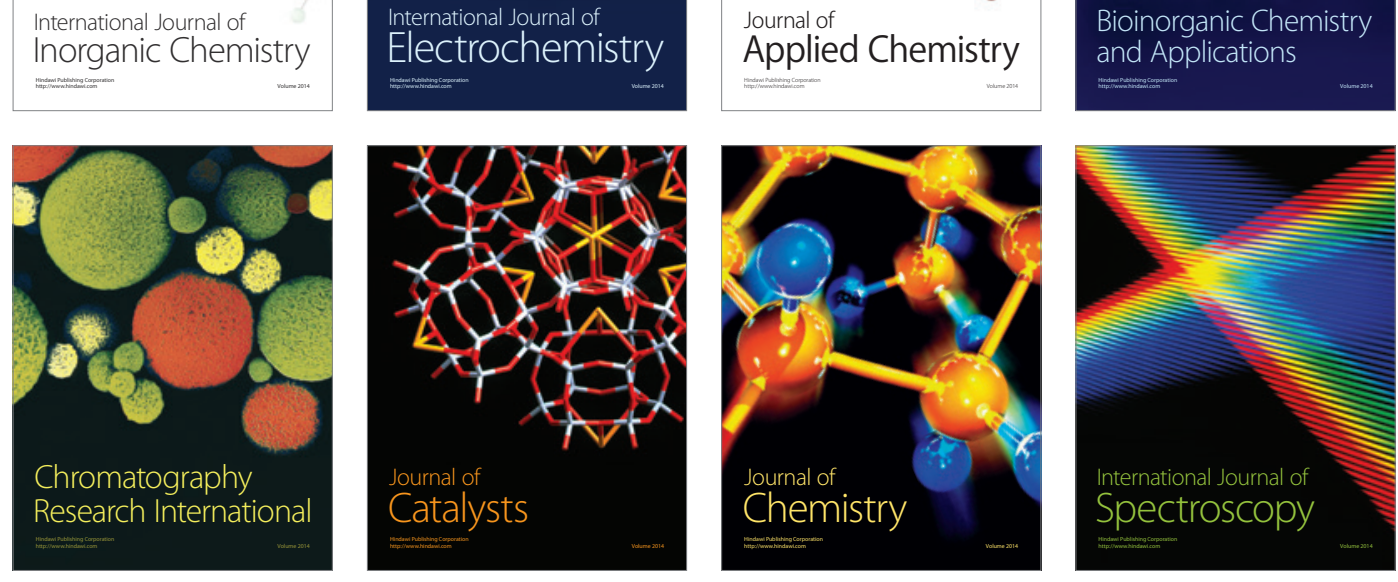